\title{
Measuring postural control in back pain patients - Center of Pressure vs. Star Excursion Balance Test
}

\author{
Hannes Zelenka, Thore-Björn Haag*, Martin Handel and Christian Schneider \\ Orthopädiezentrum Theresie, MiSpEx Research Network, Germany
}

*Corresponding author: Thore-Björn Haag, Orthopädiezentrum Theresie, Dres Schneider, Obersteiner \& Kollegen, Theresienhöhe

13A, 80339 Munich, Germany

ARTICLE INFO
Received: 幽 January 17, 2020

Published: 蔧 January 29, 2020

Citation: Hannes Zelenka, Thore-Björn Haag, Martin Handel, Christian Schneider. Measuring postural control in back pain patients - Center of Pressure vs. Star Excursion Balance Test. Biomed J Sci \& Tech Res 25(1)-2020. BJSTR. MS.ID.004144.

Keywords: Back Pain; Postural Control; Center of Pressure; Training

\section{ABSTRACT}

Purpose: To compare two different measurements of postural control and the effects on the outcomes through a training intervention including sensory motor approach.

Design: Randomized controlled trial, Re-Test design.

Methods: In a re-test design (3 weeks intervention), five different centers (of the MiSpEx consortium) investigated changes in balance ability in subjects with back pain. Primary outcomes included Single-Leg Balance-Stance for 15s (center of pressure, COP) for assessment of static postural control and the Star-Excursion-Balance-Test (SEBT) for dynamic (functional) balance ability. Changes in pain intensity were registered due to a Visual Analogue Scale (VAS) and Korff Pain Questionnaire (Korff).

Results: Summarizing retest measurements of 331 patients, COP outcomes had a low ( $\mathrm{r}=-0.17)$ but

significant correlation to SEBT-values, but were not significantly influenced by pain intensity $(p>0.05)$ in contrast to functional balance $(p<0.05, r=-0.19)$. The intervention group seemed to be more resistant to a loss of static and dynamic postural control at more intense pain after 12 weeks of regular training $(\mathrm{p}=0.023)$.

Conclusion: Although both tests were able to detect effects of regular sensory motor training, only the

functional balance task could be connected to pain intensity and disability. These findings moot a closer look at functional tests for prognosis and diagnostics of back pain and challenge the current standard.

\section{Introduction}

The assessment of postural control through a force plate in different testing conditions (e.g. normal/single- leg-stance, eyes opened/closed), is well-known and widely accepted as the 'gold-standard' (Hrysomallis, 2011), although some limitations have been documented (e.g. influence of time and day, sampling duration and frequency, [1]). Nevertheless, concerning back pain these outcomes showed to be less evident especially in the case of psycho-social components (painprocessing/-quality), stress or limitations of daily routines, which proofed to have a big influence on BP incidence. Therefore, it still remains unclear if the center of pressure is able to represent postural stability when weaknesses of responsible parameters cannot be identified correctly, although back pain proofed to result in a loss of trunk control, resulting in higher continuous basic stress for the lower back. Several authors already distinguished between the hip and an ankle strategy for balance control, being activated depending on the amount of deflection. Therefore, considering multiple interactions between physiological structures from bottom to top taking part in the process of trunk control, the ankle and its' stability seem to have the biggest impact on low deflections covered by the COP, while results of the SEBT are more likely influenced by the whole lower limb. Summarizing, it still remains unclear if an easy task (COP) is able to effectively moderate back pain (-intensity) by means of a loss in 
postural control, or if functional balance tasks (e.g. SEBT) may be more suitable. Thus, the aim of this investigation was to identify whether a functional test (like the star-excursion-balance- test) is more likely to detect psychosocial distress and changes in postural control than the COP assessment, or not.

\section{Methods}

The National Research Network for Medicine in Spine Exercise (MiSpEx, Germany) was built in 2011 with the aim to develop, evaluate and transfer a functional diagnostic as well as an intervention for the prevention and therapy of back pain patients (general population and elite athletes). More than 15 research centers with clinical or scientific background from all over Germany are participating.

\section{Trial Design}

This study is, as part of MiSpEx, designed as a multi-center randomized controlled trial, investigating an intervention for prevention and therapy of back pain (2010-2018) over a period of 12 weeks of center/homebased training (M1-M3, Figure 1). This paper is focusing the effects on static and/or dynamic postural control of a sensory motor training intervention (neuronal adaptation) and may identify, whether back pain intensity can be moderated by outcomes of COP/SEBT, or not.

\begin{tabular}{|c|c|c|c|c|c|c|}
\hline & 3 weeks & & 3 weeks & & 6 weeks & \\
\hline M1 & Center-based & M2 & Home-based & M3 & Home-based & M4 \\
\hline - COP & - Guided & $-\mathrm{COP}$ & - Self-dependent & $-\mathrm{COP}$ & - Self-dependent & $-\mathrm{COP}$ \\
\hline$-\mathrm{CS}$ & intervention & $-\mathrm{CS}$ & training planned by & $-\mathrm{CS}$ & training & $-\mathrm{CS}$ \\
\hline - Questionnaire & -3 times a week & - Questionnaire & therapists & - Questionnaire & - Regular & - Questionnaire \\
\hline - VAS & - Starting at Lvl & - VAS & - Regular & - VAS & Documentation of & - VAS \\
\hline - Anamnesis & $1 / 12$ & & Documentation of & - Anamnesis & pain intensity \& & - Anamnesis \\
\hline - Clin. Exam. & $\begin{array}{l}\text { - Individual } \\
\text { progress }\end{array}$ & & $\begin{array}{l}\text { pain intensity \& } \\
\text { stress }\end{array}$ & - Clin. Exam. & stress & - Clin. Exam. \\
\hline
\end{tabular}

Figure 1: Workflow within the multi-center.

The main study is investigating effects of a sensory motor training for a period up to 12 weeks with a follow-up of 3 months. Regarding drop-outs in the follow-up, data analysis within this paper has been reduced to $80 \%$ completion of the study (M4/5; 12 weeks of training). Nevertheless, current literature is confirming changes of neuromuscular activity already within six weeks of sensory motor training. Thus, it can be assumed that our study design is adequate to cause identifiable adaptations.

\section{Participants}

During a period of July 2013 to December 2014, 272 women (age: $38.2 \pm 14.1$ ) and 185 men (age: $38.2 \pm 12.5$ ) from Germany took part in the study. Eligibility criteria covered: age 18 to 65 , able to stand on one leg, no illness with contraindication of activity, no pregnancy, no subject of any other Mispex study and a signed consent implicating the voluntariness of participation. Measurements took place in one of five centers (Dresden, Frankfurt, Heidelberg, Munich and Potsdam), each with the same protocol administered by the central coordination based in Potsdam, Germany.

\section{Intervention}

The intervention applied can be addressed via www. mispex-network.de and consists of four different sensory-motor based exercises, each divided into 12 levels of difficulty (Figure 2). Training on unstable surface was obligate three times a week, the first three weeks within the research center and further 1.5 months at home on one's own responsibility. All participants started at the lowest difficulty regardless of their current physical fitness but were able to individually add further weights (e.g. barbells). The training process (pace, breaks) was standardized with an auditory control mechanism (signals via DVD), introduced and explained at the center and easily applicable for the use at home. Therapists confirmed the regular completion of training, while a diary needs to be filled out after every session of the home-based phase.

\section{Outcomes}

As a primary outcome, COP was defined as 'total path of the point of force application, during a single leg balance stance for 15s' on a modified Wii-board (USB-port, test frequency: $100 \mathrm{~Hz}$ ). Means of two trials for each leg (randomized; hands on hips, barefoot) were calculated. The equipment has been validated against AMTI and Kistler force plates, before. Additionally, the SEBT was assessed in accordance with frequently used protocols (BELEG Gribble), defined as the maximum reach distance in a single leg stance, normalized by leg length (so called: Composite-Score (CS); max. of three trials, hands on hips, barefoot). As a secondary outcome, 
a Visual-Analogue-Scale was consulted to interpret pain intensity during test intervals. Furthermore, Korff questionnaire (von Korff BELEG) should evaluate disability (KDis) through symptoms, pain intensity (KPain) and pain grade (KGrade) of the subjects within three parameters. Both scales were used to identify whether the static and dynamic balance tests are able to address changes in pain intensity, or not. The staff responsible for the measurements was instructed centralized, two months before study kick-off. All tests were absolved in order as they appear in the text, once each time of measurement.

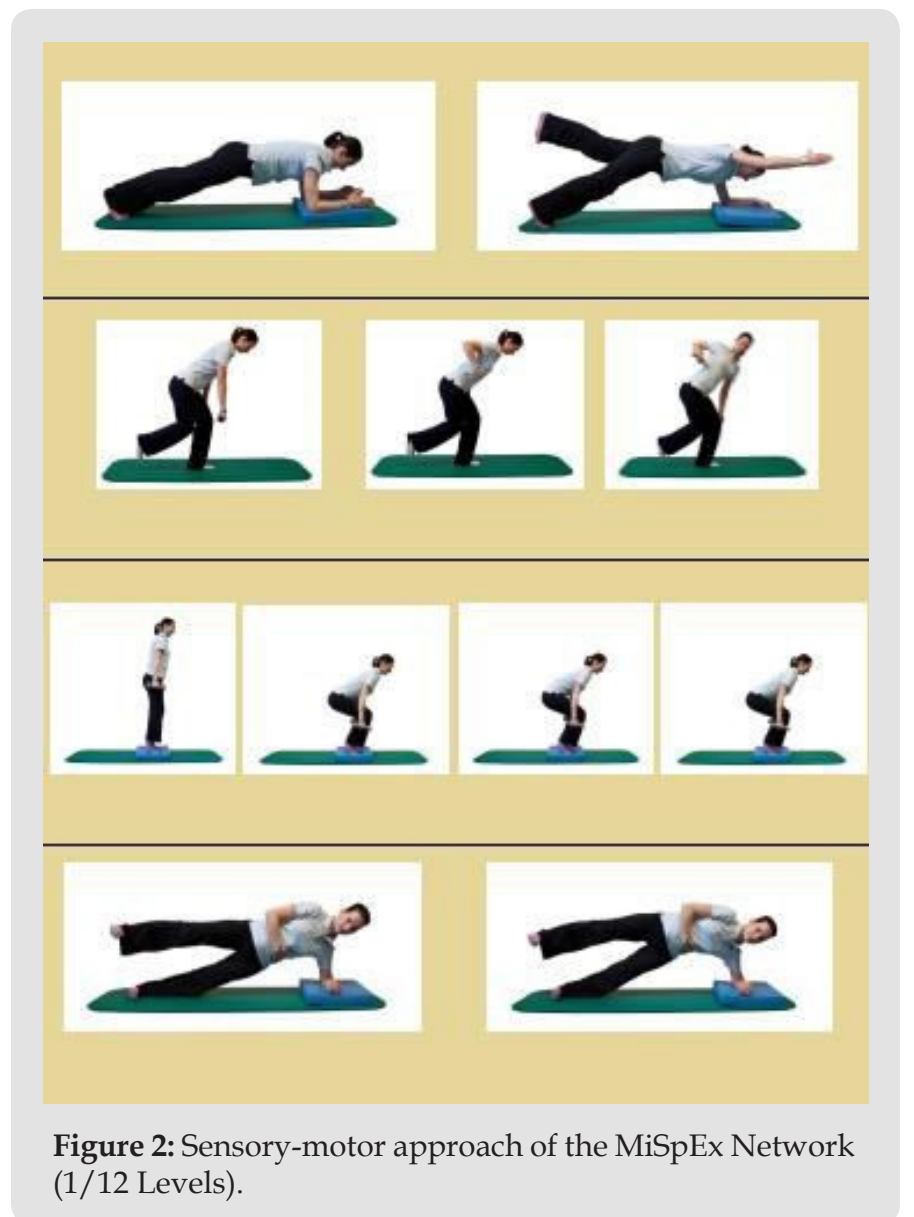

\section{Sample Size}

To achieve clinical relevance for our primary outcomes within an analysis of variance with repeated measurements, an effect size of $\mathrm{f}=0.14$ (cohen, 1988) with $\alpha=0.05$ and $1-\beta=0.8$ - regarding our pilot investigation - was determined. Assuming a drop-out rate of $30 \%$, calculations via g-power lead to a total sample size of $n=580$ for all research centers. Based on current literature, secondary outcomes were evaluated as clinical relevant within a middle effect size of $\mathrm{f}=0.25$.

\section{Randomization}

Each center assessed 145 participants for eligibility and assigned them randomly (the total of $\mathrm{N}=725$ got block randomized by Potsdam, 5 Blocks, 145 each, proportion 1:2) to one of two groups: control group ( $\mathrm{CG}$, no intervention) or intervention group (IG, 3 weeks sensory motor training). The study coordinators of each center enrolled the participants and assigned them to the interventions, based on sequentially numbered blocks given by Potsdam. 532 participants were randomized to either CG or IG, as the rest declined to participate or did not meet the inclusion criteria.

\section{Statistical Methods}

Anthropometric data was assessed during the clinical anamnesis within a standardized protocol. Normal distribution was evaluated graphically including box-, distribution- and qq-plots as well as a numerical test (shapiro-wilk-test). Correlations between variables were calculated via Pearson product moment correlations ( $r$ ), the central tendency between groups was identified via t-test and Wilcoxon-test, respectively. An analysis of variance evaluated the influence of pain on the outcomes of SEBT and COP. A post hoc analysis was conducted with G-power to evaluate the overall power of the results.

\section{Results}

Between $07 / 2013$ and 12/2014, a total of 532 participants has been randomized, allocated to the interventions and measured corresponding to the illustrated protocol (166/366 CG/IG, see CONSORT, Figure 3). 126 got lost to follow-up because of sudden inaccessibility or missing interest in further attendance. Additionally, 75 records were excluded due to missing data, resulting in a sample of $n=331$ participants (108/223 CG/IG) entering the analysis. Baseline characteristics are shown in Table 1 No significant differences could be identified between the two intervention groups, but gender specific characteristics within each. The g-power post hoc analysis, given a significance level of $\alpha=0.05$, a sample size of $n=331$ and a calculated effect size $d z=0.27 \pm 0.05$ (calculated via mean and sd of both groups), ended up in a high Power of 0.95, although group sizes are unequal.

Therefore, our study design seems qualified to detect possible associations between the groups within the outcomes, if any occur. Descriptive statistics are shown in Table 1 for all subjects. COP values significantly $(<0.05)$ improved in both groups while the CS improved highly significant, just at the second measurement, ending up in a loss of all benefits due to M3 for both groups. Within the 12 weeks of training, Korff disability and pain scores (KDis, KGrade, KPain) did improve highly significant in the intervention group $(\mathrm{p}<2.9 * \mathrm{e}-9)$ but also still significant in the CG $(\mathrm{p}=0.007)$ Table 2. The following matrix (Figure 3) illustrates all Pearson correlations between tests of balance, the VAS and Korff data at M4 within the total sample. Values of M1/M2/M3 are identical with an estimated correlation of $r=0.93$ to those of M4. 
Table 1: Baseline characteristics of the sample; ${ }^{*} p<0.05$ significant gender-specific differences within each group.

\begin{tabular}{|c|c|c|c|c|c|c|c|}
\hline Grp & Gender & Age & Height & Weight & VAS & $\mathbf{n}$ & $\mathbf{p}$ \\
\hline \multirow[b]{3}{*}{ IG } & Total & $40.8 \pm 13.0$ & $173.5 \pm 9.4$ & $73.9 \pm 14.2$ & $1.2 \pm 1.6$ & 223 & $>0.05$ \\
\hline & \& & $41.2 \pm 13.5$ & $167.9 \pm 7.0 *$ & $67.8 \pm 12.9^{*}$ & $1.36 \pm 1.6$ & 130 & \multirow{2}{*}{$<0.01$} \\
\hline & कా & $40.3 \pm 12.3$ & $181.1 \pm 6.3^{*}$ & $82.3 \pm 11.3^{*}$ & $0.97 \pm 1.6$ & 93 & \\
\hline \multirow[b]{3}{*}{ CG } & Total & $32.7 \pm 12.8$ & $172.8 \pm 8.7$ & $70.5 \pm 14.2$ & $0.9 \pm 1.6$ & 108 & $>0.05$ \\
\hline & \& & $32.4 \pm 13.5$ & $168.0 \pm 5.7^{*}$ & $63.6 \pm 9.9^{*}$ & $1.08 \pm 1.6$ & 75 & \multirow{2}{*}{$<0.01$} \\
\hline & कా & $33.3 \pm 11.8$ & $180.8 \pm 6.8^{*}$ & $81.8 \pm 12.9^{*}$ & $0.60 \pm 1.5$ & 33 & \\
\hline
\end{tabular}

Table 2: Group and date specific descriptive data for the primary and secondary outcomes.

\begin{tabular}{|c|c|c|c|c|c|c|c|c|c|c|}
\hline Grp & \multicolumn{4}{|c|}{ Intervention group | $\mathbf{n = 2 2 3}$} & \multicolumn{5}{c|}{ Control group | n=108 } \\
\hline Day & COP [mm] & CS & KDis & KGrade & KPain & COP $[\mathrm{mm}]$ & CS & KDis & KGrade & KPain \\
\hline M1 & 358.4 & 0.85 & 20.7 & 1.3 & 32.7 & 322.9 & 0.87 & 13.0 & 1.2 & 28.5 \\
\hline M2 & 346.3 & 0.87 & 14.4 & 1.2 & 27.8 & 310.9 & 0.90 & 9.8 & 1.1 & 23.6 \\
\hline M3 & 334.6 & 0.88 & 12.0 & 1.1 & 26.6 & 296.1 & 0.89 & 8.5 & 1.1 & 21.3 \\
\hline M4 & 321.3 & 0.87 & 10.4 & 1.2 & 23.4 & 297.1 & 0.89 & 7.7 & 1.2 & 23.7 \\
\hline
\end{tabular}

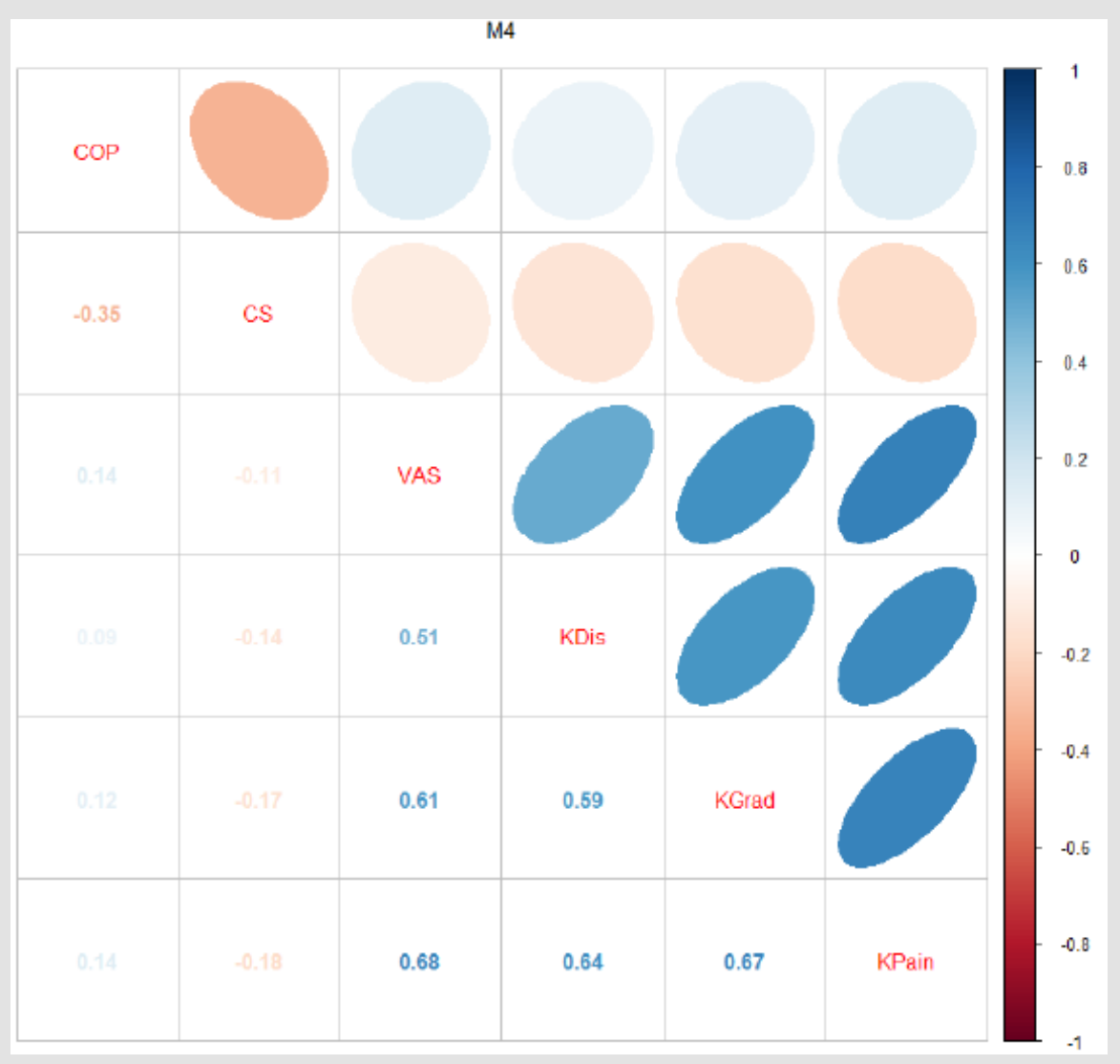

Figure 3: Correlation between the outcomes of COP, CS, VAS and the Korff questionnaire at M4.

Therefore, middle associations between functional and static balance tests could be identified ( $\mathrm{r}=-0.35 \pm 0.04)$, while the outcomes of the SEBT are significantly correlated (negatively) to the VAS, Korff pain, and disability ( $r=-0.15 \pm 0.02)$. COP seems to be slightly less influenced by pain intensity $(r=0.12 \pm 0.03)$. Figure 4 addresses the negative correlation between the center of pressure and the SEBT outcomes (upper). CS and COP are qualitatively reduced with higher pain grades (mid \& lower). High values of COP can be seen as a sign for low balance ability the way as low CS are to be interpreted. Remarkably, the intervention group seems to be more resistant to a loss of static and dynamic postural control at more intense pain after 12 weeks of regular training $(p=0.023$, Figure 5). The biggest difference can be seen after the complete training phase of 12 weeks. 

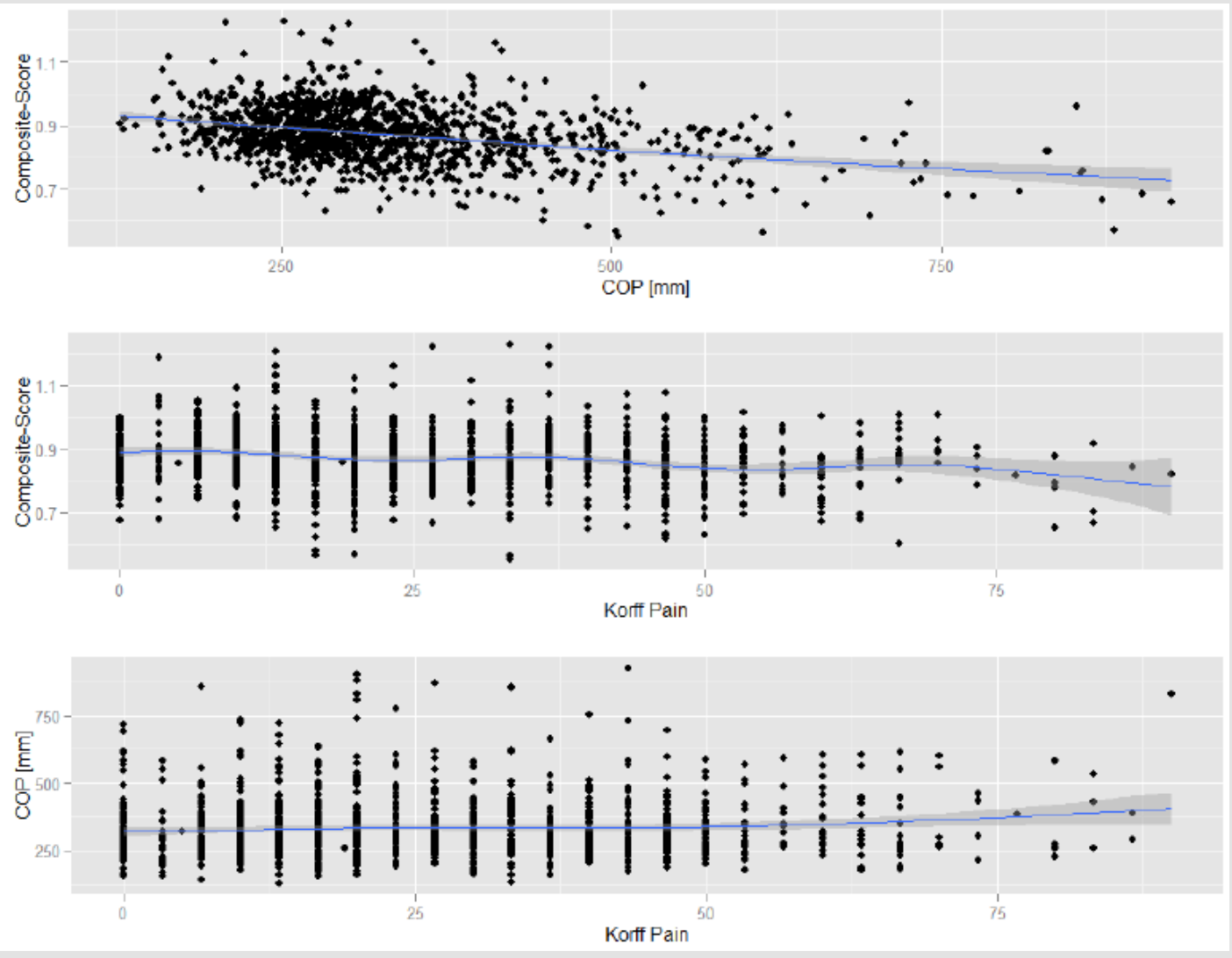

Figure 4: Overall relationship between the outcomes of COP and CS as well as Korff Pain Grade.

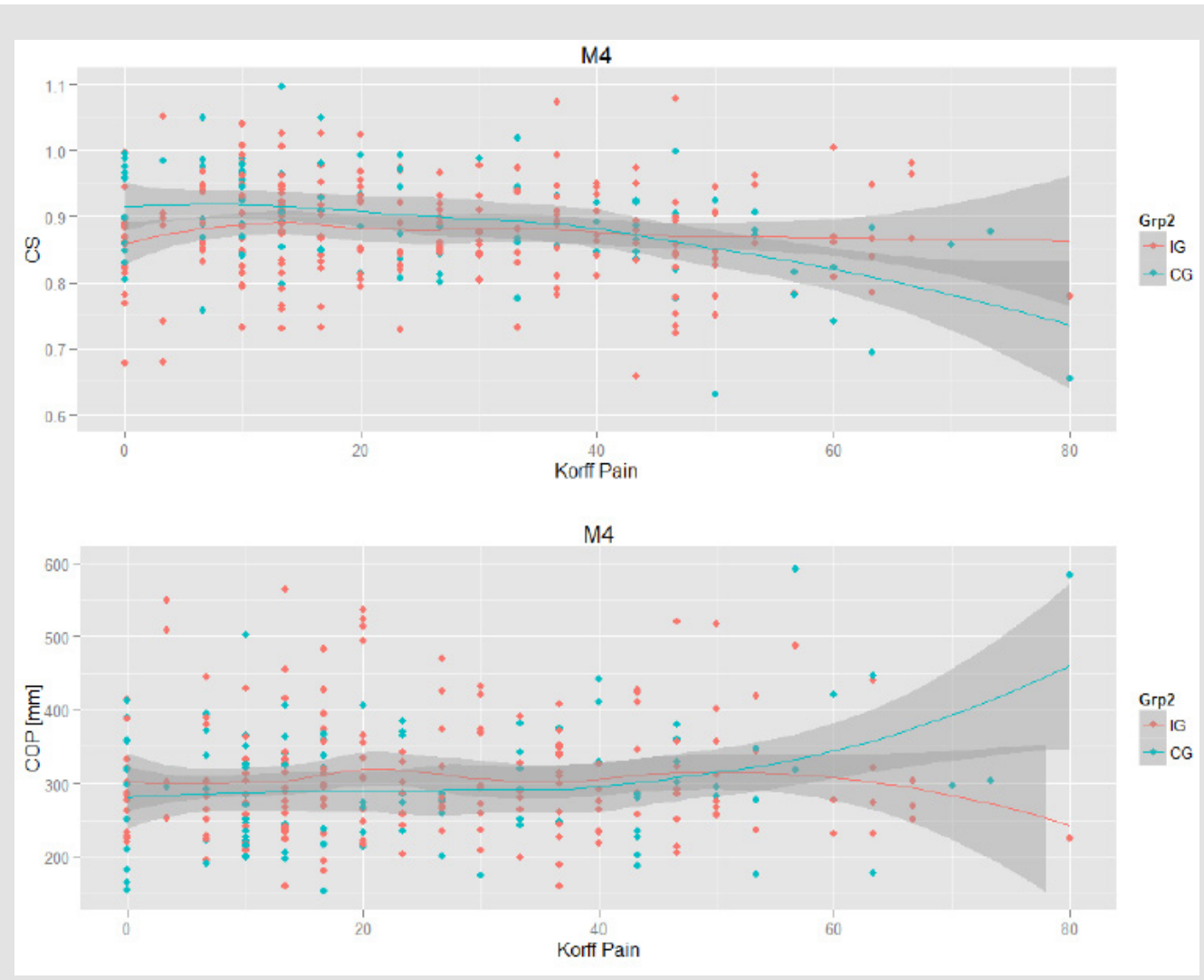

Figure 5: Illustration of the effects of the sensory-motor training intervention on resistance to a loss of postural control within high pain intensity. 


\section{Discussion}

Reviewing our results, the assessment of functional parameters seems to be more appropriate for diagnosis and preventive management of back pain patients than measuring the static postural control. Although it is mentioned that primarily daily/sport-specific movement patterns need to be considered in functional tasks (approving single and dual balance stance tests), the COP could not distinguish between a change in functionality or pain grade. In contrast to, the functional test (SEBT) showed superior effects from a diagnostic point of view. This hypothesis is also supported by psycho-social investigations, which identified limitations in motion and within the social/work context as essential factors for the evolution of back pain. Therefore, the content validity seems higher than in static balance tasks.

Furthermore, the so-called "gold-standard" seems questionable, because the main stabilization in one-leg stance tests (without external perturbation) is, verifiably, guaranteed by the ankle; whether such static balance tests are able to significantly identify back pain as a result of changes in trunk control or not, remains doubtfully. Hence, the quality may increase if methods would point closer to the trunk to ensure postural control. Diagnostics of the maximal force output of the trunk seem to be debatable against the background of a distinctive pain symptomatology and therefore are inappropriate for serious complaints. Additionally, a closer look at functional tests reveal their economic value as well: not only are most of them short-lived, but applicable without any further expensive equipment. This makes it attractive for practitioners to adapt. Nevertheless, it must be considered that functional tests mostly lack in internal validity due to an unprecise operationalization and the complex interaction between several segments of the body.

Changes in the outcome (e.g. in SEBT) must not be referred to a single cause but can be the result of several uncertainties of measurement. Thus, especially the objective evaluation of the outcomes or abortion criteria remains the main weakness. Considering that the assessment of the postural sway can be seen as a trivial task without any physical demands, it might be more suitable for patients with severe pain. Indeed, VAS proofed to be no predictor for outcome in measures of static postural control, resulting in a call for further research to support the COP as a valid tool for the assessment of postural control in back pain patients. Some limitations need to be considered while interpreting our results: first of all, center-based effects could not be controlled. To minimize potential bias, all centers followed a standardized protocol with validated hard-/software and a clear definition of inclusion criteria. Additionally, a range check and random control of $10 \%$ of the data ensured correctness.

Besides, certain investigations deal with the influence of measuring frequency and period on COP outcomes (sampling duration effect, Le Clair \& Riach, 1996; Schubert et al., 2012), advising a total of 60 s for each measurement. This is supposed to avoid interference of singular frequencies, as the main spectrum lies beneath $1 \mathrm{~Hz}$ (human body oscillation $<0.1 \mathrm{~Hz}$ ). Nevertheless, as most COP data is based on measurements of 15 to $30 \mathrm{~s}$, we decided to create comparable data. Future Research should expand measurement time to at least 60s. Summing up all arguments, functional balance control should be considered in back pain diagnostics, as it is significantly prone to pain. Especially, the SEBT, which is able to predict injuries for the lower extremities, may be used as a prognostic tool. With its' normalization within a total score and feasibility, there is less to be said against using the SEBT as an additional screening method for functional/neuronal imbalances, which may lead to back pain.

\section{Practical Applications}

This investigation recommends adding functional balance tasks to the diagnostics of back pain patients, especially for less intense pain grades. The results of our study do not support the concept of COP as a 'gold standard' for the assessment of postural control in chronic back pain patients with minor but constant pain (VAS 0-1, Korff 1). Although, it might be suitable for higher pain grades. Practitioners as well as scientists, therefore, should focus on functional tests (e.g. the SEBT) to evaluate limitations through pain symptoms.

\section{Conclusion}

This study was conducted to identify, whether current methods for the assessment of postural control may detect sensory motor training-induced changes in neuronal adaptation, or not. Furthermore, this investigation evaluated, if the existence of pain moderates the outcomes of functional or static balance control. Although both tests were able to detect effects of regular sensory motor training, only the functional balance task could be connected to pain intensity and disability (Korff). These findings moot a closer look at functional tests for prognosis and diagnostics of back pain and challenge the current 'gold standard' (COP). Nevertheless, it is advised to investigate the connection between the center of pressure and severe pain in back pain patients.

\section{Acknowledgements}

This article was conducted within the MiSpEx (National Research Network for Medicine in Spine Exercise) research consortium. This work was supported by the Federal Institute for Sport Science (BISp), Germany (ZMVI1-080102A/11-18).

\section{Statements}

\section{A. Funding Statement}

This research received no specific grant from any funding agency in the public, commercial or non-profit sectors.

B. Declaration of Competing Interests and Financial Disclosures

1) Contributors, either individually or collectively, have received no financial support, including pharmaceutical 
company support, for the authorship or promotion of contribution.

2) Contributors, either individually or collectively, have no commercial or financial involvements that might present an appearance of a conflict of interest related to the contribution.

3) Contributors, either individually or collectively, have not signed agreements with sponsors of the research reported in the contribution that places any requirements on their publication of the research findings, such as preventing them from publishing both positive or negative results or that forbids them from publishing the research without prior approval of the sponsor.

\section{Contributor Ship Statement}

All persons designated as authors qualified for authorship and their credit was based on

1) Substantial contributions to conception and design, acquisition of data, or analysis and interpretation of data

2) Drafting the article or revising it critically for important intellectual content and

3) Final approval of the version to be published. All listed authors fulfilled conditions 1,2 , and 3 .

\section{References}

1. Danneels LA, Vanderstraeten GG, Cambier DC, Witvrouw EE, De Cuyper HJ, et al. (2000) CT imaging of trunk muscles in chronic low back pain patients and healthy control subjects. European Spine Journal 9(4): 266272.

2. Finley JM, Dhaher YY, Perreault EJ (2009) Regulation of feed-forward and feedback strategies at the human ankle during balance control. Conference proceedings: ... Annual International Conference of the IEEE Engineering in Medicine and Biology Society. IEEE Engineering in Medicine and Biology Society. Annual Conference 7265-7268.

3. Gribble PA, Hertel J (2003) Considerations for Normalizing Measures of the Star Excursion Balance Test. Measurement in Physical Education and Exercise Science 7(2): 89-100.

\section{ISSN: 2574-1241}

DOI: $10.26717 / B J S T R .2020 .25 .004144$

Thore-Björn Haag. Biomed J Sci \& Tech Res

(C) This work is licensed under Creative Commons Attribution 4.0 License

Submission Link: https://biomedres.us/submit-manuscript.php
4. Haber P (2009) Leitfaden zur medizinischen Trainingsberatung Rehabilitation bis Leistungssport. Wien: Springer 3.

5. Ham YW, Kim DM, Baek JY, Lee DC, Sung PS, et al. (2010) Kinematic analyses of trunk stability in one leg standing for individuals with recurrent low back pain. Journal of Electromyography and Kinesiology 20(6): 1134-1140.

6. Hoy D, Brooks P, Blyth F, Buchbinder R (2010) The Epidemiology of low back pain. Best Pract Res Clin Rheumatol 24(6): 769-781.

7. Hrysomallis C (2011) Balance ability and athletic performance. Sports Med 41(3): 221-232.

8. Klein P, Sommerfeld P (2007) Biomechanics of the spine: basics, knowledge and questions: Elsevier Urban \& Fischer.

9. Le Clair K, Riach C (1996) Postural stability measures: what to measure and for how long. Clinical Biomechanics 11(3): 176-178.

10. Nashner LM, McCollum G (1985) The organization of human postural movements: A formal basis and experimental synthesis. Behavioral and Brain Sciences 8(1): 135-150.

11. Neuhauser H, Ellert U, Ziese T (2005) Chronic Back Pain in the General Population in Germany 2002/2003: Prevalence and Highly Affected Population Groups. Health service 67(10): 685-693.

12. Ruhe A, Fejer R, Walker B (2011) Center of pressure excursion as a measure of balance performance in patients with non-specific low back pain compared to healthy controls: a systematic review of the literature. Eur Spine J 20(3): 358-368.

13. Runge CF, Shupert CL, Horak FB, Zajac FE (1999) Ankle and hip postural strategies defined by joint torques. Gait Posture 10(2): 161-170.

14. Schiavenato M, Craig KD (2010) Pain Assessment as a Social Transaction: Beyond the "Gold Standard". 26(8): 667-676.

15. Schubert P, Kirchner M, Schmidtbleicher D, Haas CT, Engineering (2012) About the structure of posturography: Sampling duration, parametrization, focus of attention (part I) 5(9): 496.

16. van der Kooij H, Campbell AD, Carpenter MG (2011) Sampling duration effects on centre of pressure descriptive measures. Gait Posture 34(1): $19-24$

17. Von Korff M, Ormel J, Keefe FJ, Dworkin SF (1992) Grading the severity of chronic pain. Pain 50(2): 133-149.

18. Zech A, Hübscher M, Vogt L, Banzer W, Hänsel F, et al. (2010) Balance training for neuromuscular control and performance enhancement: a systematic review. J Athl Train 45(4): 392-403.

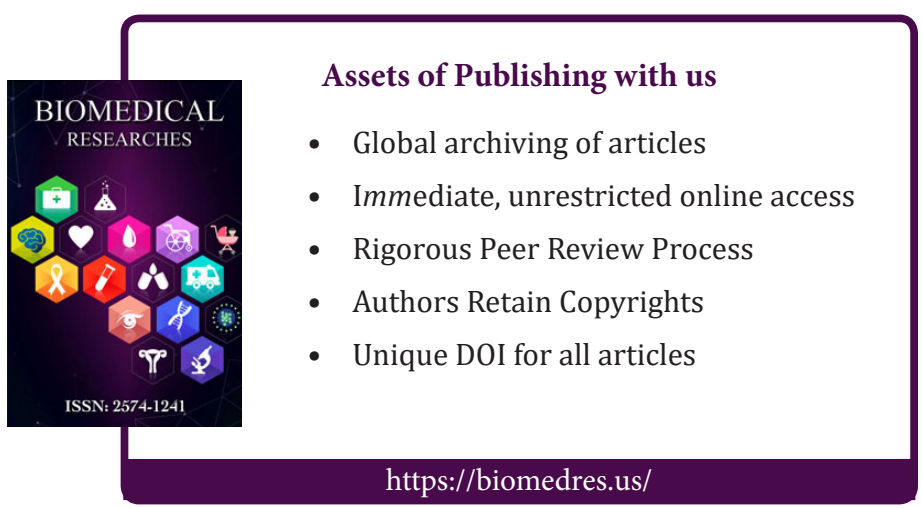

